
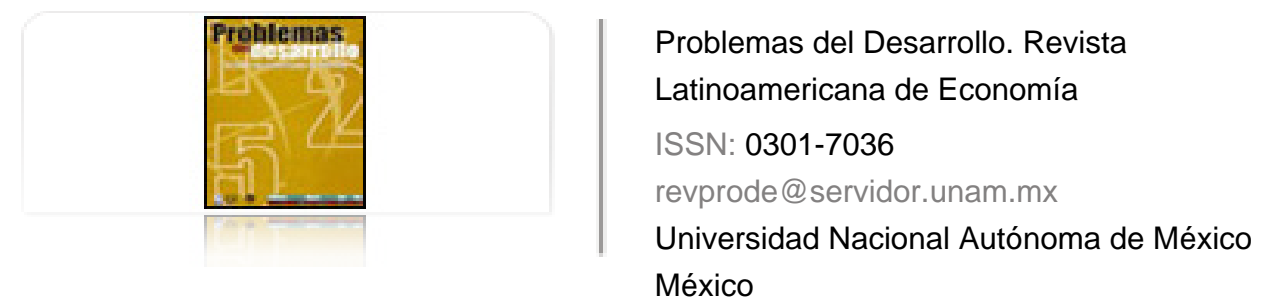

Ocegueda, Juan Manuel; Escamilla, Antonio; Mungaray, Alejandro Estructura económica y tasa de crecimiento en la frontera norte de México Problemas del Desarrollo. Revista Latinoamericana de Economía, vol. 42, núm. 164, enero-marzo, 2011, pp. 71-97

Universidad Nacional Autónoma de México

Distrito Federal, México

Disponible en: http://www.redalyc.org/articulo.oa?id=11819774005

Cómo citar el artículo

- Número completo

- Más información del artículo

Página de la revista en redalyc.org

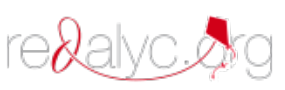

Sistema de Información Científica

Red de Revistas Científicas de América Latina, el Caribe, España y Portugal Proyecto académico sin fines de lucro, desarrollado bajo la iniciativa de acceso abierto 


\title{
ESTRUCTURA ECONÓMICA Y TASA DE CRECIMIENTO EN LA FRONTERA NORTE DE MÉXICO
}

\author{
Juan Manuel Ocegueda*, Antonio Escamilla** y Alejandro Mungaray ${ }^{* * *}$
}

Fecha de recepción: 20 de septiembre de 2010. Fecha de aceptación: 4 de noviembre de 2010.

\section{RESUMEN}

Este trabajo analiza la estructura económica de las entidades de la frontera norte de México, caracterizada por su alta concentración en un reducido grupo de industrias manufactureras exportadoras y por la alta volatilidad de su tasa de crecimiento ante las fluctuaciones de la economía mundial. Aplicando el método de Sieguel et al (1995), que vincula los modelos insumo-producto con la teoría del portafolio y utilizando la varianza de la producción regional como una proxy de la variabilidad asociada a una estructura productiva, se demuestra que entre 1993 y 2006 aumentó la volatilidad de la tasa de crecimiento en la frontera norte. Utilizando el criterio de minimización de la varianza de la producción se propone un método para seleccionar sectores que podrían contribuir a resolver este problema y a definir una estructura de producción sectorial consistente con una tasa de crecimiento estable.

Palabras clave: Estructura económica L160, análisis insumo-producto C670, desarrollo regional R110.

\section{ECONOMiC STRUCture AND THE Growth RATE ON MEXico's NORTHERN BORDER}

\section{Summary}

This study analyzes the economic structure of Mexico's northern-border states, characterized by their high concentration in a limited group of export manufacturing industries and by their highly volatile growth rates, given the fluctuations of the world economy. Applying the method used by Sieguel et al (1995), which links input-output models to portfolio theory, using the variance of regional production as a proxy for the variability associated with a productive structure, we show that between 1993 and 2006 the volatility of the growth rate increased along the northern border. Using the criterion of minimizing the variance of production, it proposes a method to select sectors that could contribute to resolving this problem and to defining a sector-specific production structure consistent with a stable growth rate.

Key words: Economic structure, input-output analysis, R110 regional development.

* Jefe de la Unidad de Presupuesto y Finanzas de la Universidad Autónoma de Baja California. Correo electrónico jmocegueda@uabc.edu.mx

** Director de Estadísticas de la Secretaría de Desarrollo Económico del Gobierno del Estado de Baja California. Correo electrónico: aescamilla@baja.gob.mx

*** Secretario de Desarrollo Económico, Secretaría de Desarrollo Económico del Gobierno del Estado de Baja California. Correo electrónico: amungaray@baja.gob.mx 


\section{STRUCTURE ÉCONOMIQUE ET TAUX DE CROISSANCE À LA FRONTIÈRE NORD DU MEXIQUE \\ Résumé}

Ce travail analyse la structure économique des entités territoriales de la frontière nord du Mexique, caractérisée par sa grande concentration dans un groupe réduit d'industries manufacturières exportatrices et par la haute volatilité de son taux de croissance devant les fluctuations de l'économie mondiale. En appliquant la méthode de Sieguel et al (1995), qui associe les modèles facteurs de production/produit à la théorie du portefeuille, et en employant la variance de la production régionale comme un proxy de la variabilité liée à une structure productive, il est démontré qu’entre 1993 et 2006 la volatilité du taux de croissance a augmenté à la frontière nord. En employant le critère de minimisation de la variance de production, il est proposé une méthode pour sélectionner des secteurs qui pourraient contribuer à résoudre ce problème et à définir une structure de production sectorielle solide avec un taux de croissance stable.

Mots clés : structure économique, analyse facteurs de production/produit, développement régional R110

\section{ESTRUTURA ECONÔMICA E TAXA DE CRESCIMENTO NA FRONTEIRA NORTE DE MÉXICO \\ Resumo}

Este trabalho analisa a estrutura econômica das entidades da fronteira norte de México, caracterizada por sua alta concentração num reduzido grupo de indústrias manufatureiras exportadoras e pela alta volatilidade da sua taxa de crescimento antes as flutuações da economia mundial. Aplicando o método de Sieguel et al (1995), que vincula os modelos de insumo-produto com a teoria do portfólio e utilizando a variância da produção regional como uma proxy da variabilidade associada a uma estrutura produtiva, se demonstra que entra 1993 e 2006 aumentou a volatilidade da taxa de crescimento na fronteira norte. Utilizando o critério de minimização da variância da produção se propóe um método para selecionar setores que poderiam contribuir para resolver este problema e a definir uma estrutura de produçáo setorial consistente com uma taxa de crescimento estável.

Palavras-chave: estrutura econômica, análise insumo-produto, desenvolvimento regional.

\section{摘要}

本文分析了在墨西哥北部边境地区的经济结构。其主要特点是出口加工 业的小范围的高度集中以及全球经济波动影响下的增长速度变化。通过 应用连接投资组合理论和投入产出模型的Sieguel方法等（1995），并 利用作为一个代理方差的区域生产结构与相关生产变异, 显示了1993 年至2006年期间在北部边境的增长率的波动性。通过利用最小方差准 则提出了一种方法来选择可以帮助解决这个问题的行业, 并确定一个能 够保持稳定增长的生产结构。 关键词：经济结构，投入产出分析，区域发展R110。 


\section{INTRODUCCIÓN}

A partir del TLCAN se ha acentuado la integración económica entre México y Estados Unidos, lo que ha contribuido a sincronizar sus respectivos ciclos económicos (Castillo, Varela y Ocegueda, 2010). Como resultado de ello, se han tenido efectos favorables en periodos de expansión de dicho país, pero también una elevada vulnerabilidad cuando ha entrado en fases de contracción o desaceleración económica. Este proceso de sincronización se ha dado con una clara diferenciación regional asociada con la posición geográfica y las vocaciones productivas de las entidades federativas, siendo las de la frontera norte las más sensibles. Las siguientes cifras ilustran esta situación: de 1993 a 2000 el pIB creció como promedio anual al 4\% en Estados Unidos, 3.6\% en México y $5.1 \%$ en la Frontera Norte (FN). De 2000 a 2002 la economía estadounidense se desaceleró cayendo su tasa de crecimiento a $1.4 \%$, lo que se reflejó de manera inmediata en México y la FN que alcanzaron tasas de 0.4 y $-0.3 \%$ respectivamente. De 2003 a 2007 Estados Unidos entró en una fase de recuperación que le permitió crecer al $2.9 \%$ anual, mientras que la economía mexicana llegó a 3.8\% y la FN a 5.2\%. Esto muestra cómo en épocas de expansión externa la producción de los estados de la FN aumenta a mayor velocidad que la nacional, mientras que en periodos de recesión mundial la caída de su producto es significativamente más pronunciada.

El objetivo de este trabajo es analizar el papel que ha jugado la concentración económica en un reducido grupo de actividades, como determinante de la alta vulnerabilidad externa y la volatilidad de la tasa de crecimiento en las entidades de la frontera norte de México (Baja California, Sonora, Chihuahua, Coahuila, Nuevo León y Tamaulipas). Asimismo, se propone un método para seleccionar sectores que podrían contribuir a resolver este problema y a definir un patrón de especialización consistente con una tasa de crecimiento más estable. La metodología de análisis se toma de Siegel et al (1995) en donde se integran los modelos de insumo-producto con la teoría del portafolio y se utiliza la varianza de la producción regional como una proxy de la inestabilidad asociada a una estructura productiva.

Estos autores plantean una estructura analítica que puede ser utilizada para estudiar la relación entre un cambio en la estructura de la demanda final y su impacto en la estabilidad del crecimiento. Así, proponen que la participación de cada sector en la demanda final puede ser considerada como una inversión individual. Este enfoque usa información sobre las variaciones porcentuales en la producción observadas en los sectores individuales y también de la interdependencia entre los sectores en la matriz de insumo-producto regional. Se calculan 
las varianzas de los cambios porcentuales observados en la demanda final en los diferentes sectores económicos y se emplean como proxies del riesgo o de la volatilidad asociada al crecimiento económico regional.

Se sostiene como hipótesis que la concentración económica en un conjunto reducido de actividades manufactureras, principalmente exportadoras, así como el abandono y contracción de otros sectores más vinculados al mercado interno, han determinado una tasa de crecimiento volátil y altamente vulnerable a los sucesos económicos externos, en la frontera norte de México. Esto sugiere un espacio para revalorizar la aportación al crecimiento que pueden hacer los sectores tradicionales proporcionando estabilidad y coadyuvando a la recuperación del mercado regional y nacional.

El documento se organiza de la siguiente forma: primero, se revisan los indicadores de desempeño económico de la FN; segundo, se analiza la estructura de la producción y el empleo y su evolución reciente; tercero, se plantean los aspectos relacionados con el método analítico y el modelo empírico utilizado; cuarto, se presentan y analizan los resultados de las estimaciones realizadas; quinto, se esbozan algunas implicaciones de política económica que se desprenden de los resultados. Finalmente, se presentan algunas reflexiones finales a manera de conclusión.

\section{DESEMPEÑO ECONÓMICO DE LA REGIÓN}

La importancia de la frontera norte dentro de la economía nacional ha venido en ascenso a lo largo de las últimas dos décadas, favorecida por el proceso de apertura comercial y las reformas estructurales que han redistribuido la producción del país, desde las entidades del centro y los viejos núcleos industriales desarrollados durante la industrialización por sustitución de importaciones, hacia las región norte y algunos otros estados que se han articulado exitosamente a la dinámica exportadora. Esto se refleja en el aporte de esta región al PIB nacional que entre 1993 y 2007 aumentó de 21.5 a 25.6\%, pero también en el mayor dinamismo ya sea que se mida por el crecimiento del PIB o del PIB per cápita, pues ambos fueron alrededor de 50\% mayores que el promedio nacional, 4.3 contra 3.0 en el primer caso y 2.5 contra 1.7 en el segundo (ver Cuadro 1 ).

Las cifras ilustran también que la región fronteriza del norte de México creció más que el país aun cuando la población y la PEA se incrementaron a tasas más altas, lo cual podría haber minado el aumento del ingreso per cápita. Esto significa que paralelamente creció el producto por trabajador a tasas superiores al promedio nacional, probablemente impulsado por reasignaciones de recursos 
Cuadro 1. Indicadores de crecimiento y desempeño económico en las entidades de la Frontera Norte

\begin{tabular}{|c|c|c|c|c|c|c|c|c|c|c|}
\hline \multirow[b]{2}{*}{ ENTIDADES } & \multicolumn{2}{|c|}{$\begin{array}{c}\text { PIBi/PIBn } \\
(\%)\end{array}$} & \multirow{2}{*}{$\begin{array}{l}\text { TCA PIB } \\
1993- \\
2007\end{array}$} & \multirow{2}{*}{$\begin{array}{c}\text { TCA PEA } \\
1993- \\
2007\end{array}$} & \multirow{2}{*}{$\begin{array}{c}\text { TCA POB } \\
1993- \\
2007\end{array}$} & \multicolumn{2}{|c|}{$\begin{array}{c}\text { PEA/POB } \\
(\%)\end{array}$} & \multirow{2}{*}{$\begin{array}{c}\text { TCA } \\
\text { PIB/POB } \\
1993- \\
2007\end{array}$} & \multirow{2}{*}{$\begin{array}{c}\text { TCA } \\
\text { PIB/PEA } \\
1993- \\
2007\end{array}$} & \multirow{2}{*}{$\begin{array}{c}\text { TCA } \\
\text { PEA/POB } \\
1993- \\
2007\end{array}$} \\
\hline & 1993 & 2007 & & & & 1993 & 2007 & & & \\
\hline$a C_{0}$ & 2.8 & 3.4 & 4.5 & 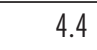 & . & 37.3 & 43.0 & 1.2 & 0.1 & 1.1 \\
\hline Coahuil & 2.9 & 3.7 & 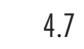 & 2.8 & 1.4 & 35.1 & 42.2 & 3.3 & 1.9 & 1.4 \\
\hline Chihuahua & 3.9 & 4.6 & 4.2 & 3.0 & 1.5 & 35.0 & 42.8 & 2.7 & 1.2 & 1.5 \\
\hline Nuevo L & 6.4 & 7.7 & 4.3 & 3.5 & 1.7 & 36.4 & 46.2 & 2.6 & 0.8 & 1.8 \\
\hline Sonora & 2.6 & 2.9 & 3.8 & 2.3 & 1.5 & 35.8 & 39.8 & 2.3 & 1.5 & 0.8 \\
\hline Tamaulip & 2.8 & 3.3 & 4.2 & 3.4 & 1.7 & 35.0 & 44.5 & 2.5 & 0.8 & 1.7 \\
\hline Frontera $\mathrm{N}$ & 21.5 & 25.6 & 4.3 & 3.3 & 1.8 & 35.8 & 43.1 & 2.5 & 1.0 & 1.5 \\
\hline Nacional & 100 & 100 & 3.0 & 2.7 & 1.3 & 34.3 & 42.0 & 1.7 & 0.3 & 1.4 \\
\hline
\end{tabular}

Fuente: Estimaciones propias con datos de INEGI y CONAPO.

hacia sectores de alta y mediana productividad, aunque ello no obedece a un proceso homogéneo que se haya extendido a toda la región. Asimismo, la tasa de actividad económica medida por la razón РЕA/РОВ aumentó de 35.8\% en 1993 a $43.1 \%$ en 2007, es decir, la gente que se incorporó a los mercados laborales se incrementó a una tasa más acelerada que la población total, si bien en este rubro los movimientos fueron muy cercanos a los nacionales. En conjunto la FN arroja buenos resultados comparados con el promedio observado en el país, tanto en materia de crecimiento de la productividad como del empleo.

Es importante precisar que el desempeño de las entidades norteñas no fue homogéneo en el periodo analizado. Coahuila es claramente el estado líder con una tasa de crecimiento anual de 3.3\%, seguida por Chihuahua y Nuevo León con tasas de 2.7 y 2.6, respectivamente. Baja California y Sonora son las entidades con peor desempeńo, la primera con una tasa inferior a la media nacional, $1.2 \%$ contra $1.7 \%$, y la segunda con una tasa menor al promedio de la región, $2.3 \%$ contra $2.5 \%$. Si se desglosa el crecimiento del PIB per cápita en sus componentes se observan distintas tendencias; por ejemplo, en Coahuila, Sonora y Chihuahua los componentes productividad y tasa de actividad muestran un crecimiento más o menos equilibrado, situación que difiere de la observada en Nuevo León, Tamaulipas y Baja California donde el componente tasa de actividad fue significativamente más dinámico. Una interpretación de estos hechos es que el primer grupo basó parcialmente su crecimiento en el incremento del producto por trabajador, mientras que el segundo privilegió una estrategia de expansión acelerada del empleo que mantuvo estancada la productividad. 
La evolución del PIB per cápita en el periodo generó cambios importantes en la distribución del ingreso por regiones y entidades federativas, ampliando la brecha entre la FN y el resto del país que pasó de 1.28-1 en 1993, a 1.40-1 en 2007. Al interior de la FN, Nuevo León se sostuvo como el estado más rico incrementando la ventaja sobre Baja California y Sonora; Coahuila se reposicionó favorablemente al pasar de la cuarta a la segunda posición de acuerdo con el ingreso por habitante, mientras que Baja California cayó del segundo al quinto lugar. Tamaulipas se mantuvo como la entidad de menor ingreso a lo largo de todo el periodo. El análisis muestra que gran parte de estos movimientos se gestaron a partir de 2001, año de crisis en Estados Unidos, y se relacionan con los impactos diferenciados de dicha crisis y con las distintas capacidades de recuperación por parte de las entidades federativas (ver Gráfica 1).

Gráfica 1. PIB per cápita 1993-2007

(pesos de 1993)

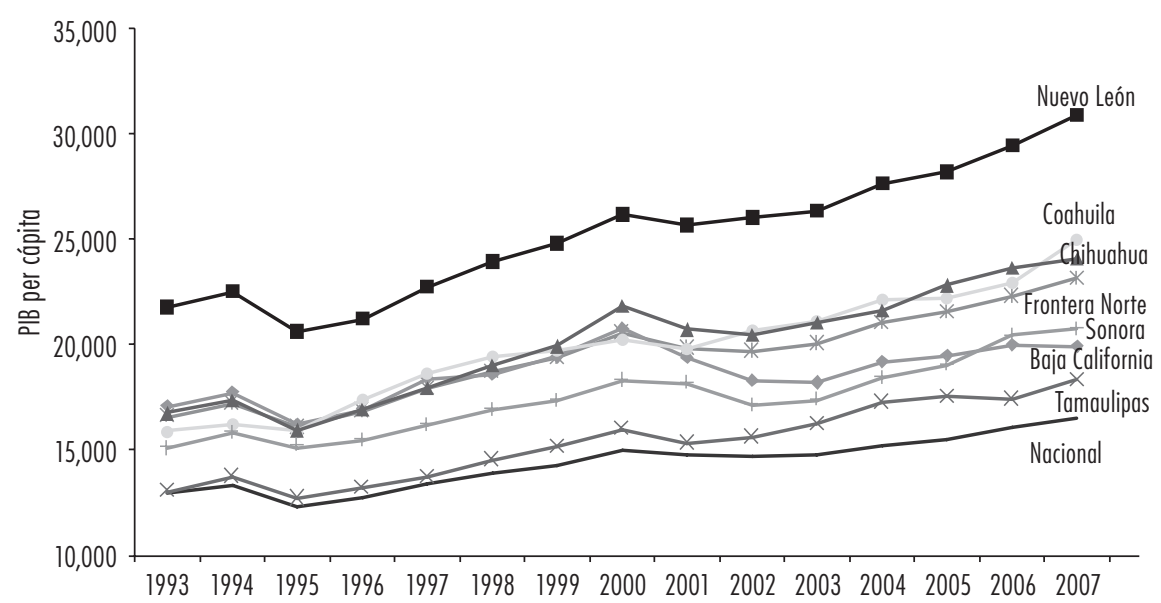

Llama la atención el caso de Baja California en donde el lento dinamismo del PIB per cápita coincide con un elevado dinamismo del PIB agregado, cuya tasa de crecimiento fue la segunda más grande en la región y la cuarta a nivel nacional. Esto se explica por su explosiva dinámica demográfica debido a los intensos flujos migratorios provenientes del resto del país, lo que abre una interrogante en términos de si el lento crecimiento a que se hace referencia es en realidad un signo de mal desempeño económico, pues resulta obvio que la fuerte 
atracción migratoria refleja la percepción positiva que tiene la gente en torno a las oportunidades que ofrece esta entidad. A favor de esta idea se puede señalar la baja tasa de desempleo que mantiene y que figura como una de las menores en la región y en el país, a pesar del rápido aumento de la PEA.

Una de las consecuencias de la integración económica de México a los mercados internacionales, impulsada desde mediados de los 1980 a través de la apertura comercial y consolidada con la firma del TLCAN y otros tratados comerciales similares que se firmaron posteriormente con otros países, fue que sincronizó el ciclo económico nacional con el del resto del mundo, particularmente con el de Estados Unidos. Ello significa que la economía mexicana se volvió más vulnerable a los choques externos y más sensible a los fenómenos económicos extranacionales, lo cual puede generar impactos positivos cuando hay expansión mundial, pero también efectos negativos cuando se tienen periodos recesivos. Dicho proceso se manifiesta a través de una mayor dependencia en el crecimiento del PIB con respecto al dinamismo de las exportaciones, lo que a su vez determina otros desequilibrios de carácter regional al interior del país, pues es conocido que las plataformas exportadoras están localizadas en pocos estados: el DF y las entidades de la FN, principalmente.

La elevada dependencia de los estados fronterizos del norte de México con respecto a lo que ocurre en Estados Unidos no es un fenómeno nuevo. Tradicionalmente esta región ha mantenido lazos muy estrechos de interdependencia económica con sus estados vecinos de dicha nación, lo que les ha permitido sobrevivir en un contexto de intensa competencia. Así, la región se ha beneficiado frecuentemente con las fases expansivas de la economía estadounidense que se traducen en un crecimiento superior al del resto del país, pero en algunas ocasiones también, cuando a Estados Unidos le ha ido mal, se han dejado sentir sus efectos contractivos sobre la actividad económica regional con una intensidad mayor al promedio nacional. Al no haber datos disponibles de PIB estatal para 2009, año en que se ha sufrido una de las crisis económicas internacionales más profundas de las últimas décadas, el ejemplo más cercano que ilustra este fenómeno es la desaceleración estadounidense de 2000-2001 asociada parcialmente con los eventos terroristas de septiembre de ese año.

Si se observa la Gráfica 2 se puede apreciar, primero, que efectivamente, quitando la crisis de 1995 que tuvo sus orígenes en México, los ciclos de ambos países tienden a sincronizarse probablemente con algún retraso temporal que llega a ser menor a un año y que es mucho más palpable en el resto del país que en la frontera norte. Segundo, tanto las caídas como los repuntes de la tasa de crecimiento en respuesta al ciclo norteamericano son mucho más pronunciados en la FN que en el resto del país. Tercero, cuando las crisis tienen origen nacional 
como en 1995, la economía de la FN cae menos y se recupera más rápido, aunque esta última característica se repite también cuando las crisis son de naturaleza externa.

Gráfica 2. Tasas de crecimiento del PIB 1993-2007

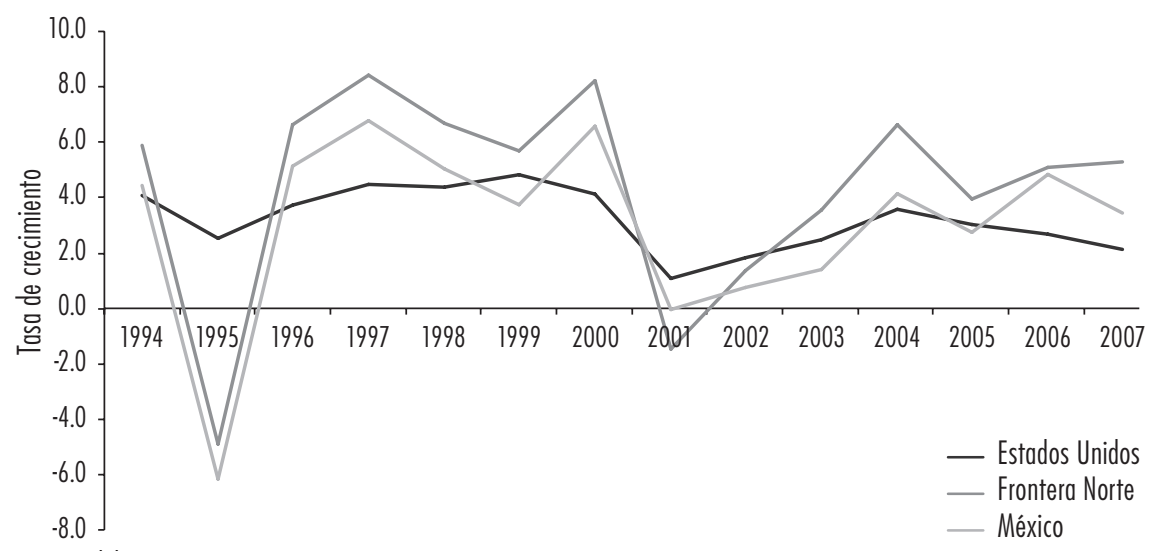

Fuente: Elaboración propia.

\section{ESTRUCTURA PRODUCTIVA}

La estructura económica de la frontera norte tiene una fuerte orientación hacia las actividades manufactureras y terciarias que en conjunto contribuyen con el $82.5 \%$ del PIB, predominando entre estas últimas las relacionadas con servicios comunales, sociales y personales; comercio, restaurantes y hoteles; y servicios financieros, aportando respectivamente el 19.6, 18.2 y 12.8\%. Las manufacturas con el $24.6 \%$ se ubican en primer lugar si se toma como referencia la clasificación por grandes divisiones. En cuanto al dinamismo sectorial entre 1993 y 2007 se observa que las actividades de transporte y comunicaciones, comercio y manufacturas son las que se expanden mas rápidamente alcanzando tasas de $7.4,4.8$ y 4.7\%, mientras que los sectores agropecuario, construcción y el resto de los servicios, con tasas de 1.8, 2.0 y 2.7 se sitúan por debajo de la media regional. Estas cifras implican que el primer grupo elevó su contribución al producto y el segundo sufrió una contracción relativa con pérdida de participación en el рів (ver Cuadros 1 у 2).

El análisis por entidades arroja que en materia de crecimiento sectorial en actividades dinámicas, Coahuila, Tamaulipas y Baja California ejercen el liderazgo en la industria manufacturera con tasas de 5.7, 5.1 y 5.0\% anual en el periodo 
Cuadro 2. Participación de las actividades económicas dentro del PIB en la Frontera Norte, 2007 (\%)

\begin{tabular}{|l|r|r|r|r|r|r|r|}
\hline & $\begin{array}{c}\text { Frontera } \\
\text { Norte }\end{array}$ & $\begin{array}{c}\text { Baja } \\
\text { California }\end{array}$ & Coahuila & Chihuahua & $\begin{array}{r}\text { Nuevo } \\
\text { León }\end{array}$ & Sonora & Tamaulipas \\
\hline Agropecuaria (I) & 4.15 & 3.11 & 2.63 & 6.00 & 0.76 & 8.68 & 3.71 \\
\hline Minería (II) & 2.47 & 0.23 & 2.41 & 0.36 & 1.68 & 2.29 & 7.87 \\
\hline Manufacturas (III) & 24.63 & 22.54 & 41.20 & 26.23 & 24.08 & 20.25 & 13.48 \\
\hline Construcción (IV) & 9.11 & 9.44 & 5.90 & 7.27 & 10.14 & 7.68 & 14.20 \\
\hline Electricidad, gas y agua (V) & 1.80 & 2.18 & 1.54 & 1.29 & 1.19 & 1.59 & 3.03 \\
\hline $\begin{array}{l}\text { Comercio, restaurantes y } \\
\text { hoteles (VI) }\end{array}$ & 18.16 & 20.70 & 13.85 & 18.40 & 18.09 & 19.89 & 18.00 \\
\hline $\begin{array}{l}\text { Transporte, almacenamiento } \\
\text { y comunicaciones (VII) }\end{array}$ & 7.34 & 7.22 & 7.62 & 4.83 & 8.65 & 6.20 & 9.50 \\
\hline $\begin{array}{l}\text { Servs. financieros, seguros } \\
\text { y bienes inmuebles (VIII) }\end{array}$ & 12.77 & 13.26 & 8.66 & 13.18 & 15.23 & 12.87 & 13.39 \\
\hline $\begin{array}{l}\text { Servs. comunales, sociales } \\
\text { y personales (IX) }\end{array}$ & 19.57 & 21.32 & 16.19 & 22.44 & 20.18 & 20.55 & 16.82 \\
\hline PIB agregado & 100.00 & 100.00 & 100.00 & 100.00 & 100.00 & 100.00 & 100.00 \\
\hline
\end{tabular}

Fuente: Elaboración propia con datos tomados de INEGI, www.inegi.org.mx

1993-2007. Baja California, Chihuahua y Sonora destacan en el transporte y las comunicaciones con tasas de 8.4, 8.4 y 7.7 respectivamente, en todos los casos superiores al promedio de la región. En actividades de corte más tradicional como la minería, Nuevo León y Tamaulipas puntean logrando crecer al 7.9 y $7.8 \%$, mientras que Nuevo León y Chihuahua sobresalen en la construcción con tasas de 5.3 y 4.3, y Chihuahua y Sonora en el comercio con tasas de 5.5 y $5.4 \%$.

Las cifras disponibles sobre la evolución de la ocupación en México dan cuenta de un aumento en la contribución de la frontera norte a la ocupación nacional, de 17.4 a $18.5 \%$ entre 1996 y 2008, determinado por un crecimiento superior al promedio nacional, $2.6 \%$ contra $2.1 \%$. Ello fortalece el posicionamiento de esta región como fuente de oportunidades para muchos mexicanos que son expulsados de los mercados laborales en sus estados de origen. Un examen más puntual considerando el dinamismo con que se expande la ocupación, permite establecer que son 4 de los 6 estados que conforman la región los que explican este resultado con un desempeño superior al registrado en el conjunto del país, siendo Coahuila y Sonora los que no alcanzan este nivel. Baja California se erige como el estado con mayor capacidad para crear empleos con una tasa de $4.1 \%$ anual que prácticamente duplica a la nacional que fue de $2.1 \%$, tal como ya se había señalado en párrafos anteriores (ver Cuadro 3). 
Cuadro 3. Evolución de la ocupación en las entidades de la Frontera Norte de México 1996-2008 (\%)

\begin{tabular}{lrrrcc}
\hline \multicolumn{1}{c}{ ENTIDADES } & 1996 & $\begin{array}{r}\text { Ocupai/ } \\
\text { ocupan }\end{array}$ & 2008 & $\begin{array}{c}\text { Ocupai/ } \\
\text { ocupan }\end{array}$ & $\begin{array}{c}\text { TCA } \\
\text { anual }\end{array}$ \\
\hline Baja California & 811,478 & 2.4 & $1,315,572$ & 3.0 & 4.1 \\
Coahuila & 830,080 & 2.4 & $1,006,380$ & 2.3 & 1.6 \\
Chihuahua & $1,054,966$ & 3.1 & $1,384,473$ & 3.2 & 2.3 \\
Nuevo León & $1,413,049$ & 4.2 & $1,944,916$ & 4.5 & 2.7 \\
Sonora & 811,606 & 2.4 & $1,009,741$ & 2.3 & 1.8 \\
Tamaulipas & 995,043 & 2.9 & $1,333,280$ & 3.1 & 2.5 \\
Frontera Norte & $5,916,222$ & 17.4 & $8,054,360$ & 18.5 & 2.6 \\
Nacional & $33,968,601$ & 100.0 & $43,517,182$ & 100.0 & 2.1 \\
\hline
\end{tabular}

Fuente: INEGI, Encuesta Nacional de Ocupación y Empleo, varios años.

En cuanto a la dinámica de reasignación de recursos entre 1996 y 2008 se pueden percibir algunos cambios que permiten identificar tendencias de especialización y desespecialización dependiendo de si la contribución del estado al total nacional tiende a crecer o a caer. En el primer caso se tiene a la construcción, el comercio, los servicios profesionales y los servicios de carácter social, que serían áreas en donde el empleo ha aumentado en mayor proporción que en el país. En el segundo caso se agrupan las actividades agropecuarias; extractivas incluyendo a la industria eléctrica; restaurantes y servicios de alojamiento; servicios diversos; y actividades relacionadas con el gobierno y organismos internacionales. Las manufacturas, el transporte y las comunicaciones integran un tercer grupo caracterizado por una situación de estabilidad, lo cual significa que mantienen prácticamente la misma participación entre 1996 y 2008.

$\mathrm{Al}$ nivel de los estados y con una perspectiva dinámica las tendencias de especialización parecen moverse de la siguiente manera: todos, excepto Baja California, tienden a desespecializarse en las actividades agropecuarias, algo similar ocurre con las industrias extractiva y eléctrica aunque en este caso las excepciones son Nuevo León y Tamaulipas. En construcción, servicios profesionales y servicios sociales, la tendencia observada es de especialización en las 6 entidades, mientras que en las manufacturas para tener un resultado similar debe excluirse a Coahuila y Chihuahua. En el comercio se tiene especialización en tres entidades (BC, CHIH y TAM) y en tres no (COA, NL y SON), mientras que en res-

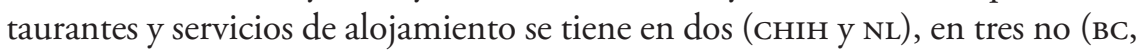
SON y TAM) y una permanece estable (COA). En transporte y comunicaciones la 
especialización avanza en Baja California, Nuevo León y Sonora y retrocede en Coahuila, Chihuahua y Tamaulipas. En servicios diversos únicamente avanza en Baja California y Sonora retrocediendo en el resto de las entidades. Finalmente, la participación del empleo estatal dentro del nacional aumenta en Coahuila y Nuevo León y disminuye en los otros estados.

$\mathrm{Al}$ interior de los estados, considerando 2008 como año de referencia, se observan los siguientes patrones de comportamiento: la industria manufacturera es la que mayor peso relativo tiene en la composición de la ocupación estatal, con excepción de Sonora y Tamaulipas, en donde el comercio ocupa dicha posición. A su vez, en estas entidades las manufacturas alcanzan el segundo lugar mientras que el comercio se ubica en esta misma posición en las cuatro entidades restantes. El tercer sitio en importancia corresponde a servicios diversos, excepto en Chihuahua donde es para el sector agropecuario. La cuarta actividad que más contribuye al empleo es servicios sociales en Baja California y Coahuila, construcción en Chihuahua y Tamaulipas, servicios profesionales en Nuevo León y las actividades agropecuarias en Sonora.

Si se analizan los cambios en el empleo sectorial de los estados, los sectores que mayor avance registran son la construcción, el comercio y los servicios profesionales; por su parte los que se contrajeron fueron los sectores agropecuario y manufacturero, industria extractiva y eléctrica, servicios diversos, y gobierno y organismos internacionales. Este comportamiento se reproduce a nivel nacional dejando ver que se trata de una tendencia más general, probablemente de alcance internacional.

\section{ASPECTOS METODOLÓGICOS}

En este apartado se exponen los elementos metodológicos de un conjunto de ejercicios que muestran la relación existente entre concentración de la actividad económica y volatilidad de la tasa de crecimiento. Se adopta la metodología propuesta por Siegel et al (1995) vinculando los modelos de insumo-producto con la teoría del portafolio para medir la relación entre un nivel de concentración o diversidad de la actividad productiva, un proceso de diversificación y la estabilidad de la tasa de crecimiento. ${ }^{1}$ La hipótesis implícita es la de una relación

1 El término diversidad se refiere a la variedad sectorial en la composición de una estructura económica, en un momento en el tiempo, consecuentemente se trata de un concepto de naturaleza estática. La diversificación se entiende como un proceso de cambios en el nivel de diversidad sectorial que alude una transformación dinámica en el tiempo. 
positiva entre diversidad productiva y estabilidad, y entre diversificación y aumento de la estabilidad, lo cual es consistente con la experiencia internacional en la que estructuras económicas diversificadas suelen asociarse con trayectorias de crecimiento estables (Lucas, 1988; Koren y Tenreyro, 2005).

Se hace una analogía entre las participaciones de cada sector en la demanda final y las inversiones individuales, en donde el total de sectores puede verse como un portafolio regional. El nivel de estabilidad o inestabilidad del crecimiento económico puede obtenerse como una función directa de la estructura económica regional, calculando la varianza de los cambios observados en la tasa de crecimiento de la demanda final en los diferentes sectores económicos, y esta variable puede emplearse como una proxy del riesgo o de la volatilidad. Por su parte, el crecimiento promedio de la demanda final de cada sector puede utilizarse como una proxy del rendimiento esperado (Siegel et al, 1995; Conroy, 1975).

De acuerdo con la estructura básica del modelo de insumo-producto se tiene:

$$
X=(I-A)^{-1} F
$$

donde:

$X$ es un vector de valores brutos de producción sectorial, cuya dimensión es $N^{*} 1$; I es la matriz identidad, de dimensión $N^{*} N ; A$ es la matriz de coeficientes técnicos de insumo-producto, dimensión $N^{*} N ;(I-A)^{-1}$ es la matriz inversa de Leontief (o matriz de multiplicadores de producción) y su dimensión es $N^{*} N$; $F$ es un vector de demanda final exógena, cuya dimensión es $N^{*} 1$.

Suponiendo que la demanda final exógena determina el desempeño de una economía, se puede modelar el crecimiento esperado y la varianza en la producción regional.

El modelo insumo-producto de la ecuación (1) en notación compacta es: ${ }^{2}$

$$
X=R F
$$

donde:

$R$ es la matriz multiplicador de la producción (o inversa de la matriz de Leontief)

2 Se toma como base la notación empleada por Siegel et al (1995). 
El valor esperado de la producción regional puede ser expresado como:

$$
E[X]=R E[F]
$$

donde:

$E[X]$ es el vector del valor esperado de los valores brutos de la producción regional por sector. Su dimensión es $N^{*} 1 ; E[F]$ es el vector de dimensión $N^{*} 1$, del nivel esperado de la demanda final exógena por sector. El valor esperado de la producción total regional es la suma de la columna del vector $E[X]$.

El crecimiento esperado en la producción regional por sector puede ser expresado como:

$$
E[\Delta X]=R\left[F_{\mathrm{t}+1}\right]-R E\left[F_{\mathrm{t}}\right]
$$

donde:

$E[\Delta X]$ es el crecimiento esperado en la producción sectorial por periodo de tiempo $t$ a $t+1$. El cambio total esperado en el crecimiento en la producción regional $E$, es la suma de la columna de $E[\Delta X]$.

Con los modelos de insumo-producto se puede hacer análisis de impacto, utilizando las ecuaciones (3) y (4), en las cuales se asume que la matriz multiplicadora de la producción es fija para el periodo de análisis. La varianza de la producción regional puede ser expresada como:

$$
V=V X]=W R C O V\left[F_{i}, F_{j}\right] R^{T} W^{T}
$$

donde:

$V[X]$ es la varianza en el crecimiento de la demanda final; $R$ es la inversa de la matriz de Leontief; $W_{\mathrm{i}}$ es la participación del sector i en la demanda final exógena $\left(0 \leq W_{\mathrm{i}} \leq 1, \sum W_{\mathrm{i}}=1\right) ; C O V\left[F_{\mathrm{i}}, F_{\mathrm{j}}\right]$ es la matriz de varianza-covarianza de la tasa de crecimiento observada en la demanda final exógena con una dimensión de $N^{*} N$; $R^{T}$ es la inversa de la matriz de Leontief transpuesta (Siegel et al, 1995).

Dado lo anterior, podemos minimizar la varianza del crecimiento sectorial y el resultado permite encontrar la estructura de la demanda final que: 1 ) con un nivel de volatilidad deseado en el crecimiento económico de la región, maximiza el crecimiento en el valor bruto de su producción (VBP) o, alternativamente, 2) con un nivel de crecimiento esperado en el vBP, minimiza la volatilidad. La minimización de la varianza en el caso de un portafolio pequeño se puede realizar aplicando una función de Lagrange (Haugen, 1997), sin embargo, puesto que 
en esta investigación se trabaja con una matriz agregada a 17 sectores, se hace necesaria la utilización de un software computacional. ${ }^{3}$

El modelo empírico se estructura partiendo de la matriz de insumo producto regional construida por Dávila (2002) para el estado de Coahuila, y se realiza el mismo procedimiento para cada entidad de la frontera norte y para la región en su conjunto. Las matrices nacionales que sirven de insumo para este ejercicio fueron calculadas a nivel nacional para los años 1993, 1996, 2000 y 2003, por la empresa Consultoría Internacional Especializada. El método empleado por Dávila (2002) fue formulado por Flegg et al (1995) y modificado por Flegg y Webber (1997). Otros trabajos de regionalización de matrices de insumo producto utilizan el método de encuestas parciales, que permite obtener parte de la información directamente de las unidades productivas (Fuentes, 2005).

Una vez elaborado el conjunto de matrices para las entidades de la frontera norte, se aplica el método de Sieguel et al (1995), descrito anteriormente, utilizando el valor bruto de la producción (VBP) del periodo 1993-2006 con un nivel de desagregación a 17 sectores. Esto permite cuantificar la varianza del vBP en cada entidad de la frontera norte de México y con ella tener una medida de la variabilidad de la producción. Asimismo, se puede estimar el crecimiento esperado del vвp para el ańo $t+1$ y minimizar la varianza del vip asociada con una meta de crecimiento y con una determinada composición de la producción. De esta manera, es posible identificar la estructura sectorial, utilizando para ello la composición porcentual de la demanda final, consistente con una variabilidad mínima de la tasa de crecimiento económico.

\section{ANÁLISIS DE RESULTADOS}

Las estimaciones de la varianza utilizando matrices de insumo-producto para los años 1993 y 2003 por estado y para la región en su conjunto, se reportan en los Cuadros 4 y 5. En la última fila de cada cuadro se presenta el coeficiente de correlación $(R)$ entre la varianza observada y el índice de concentración/especialización de Hirschman-Herfindall (HHI), para la gran división de manufacturas.

3 En este trabajo se utiliza el paquete de cómputo Excel, sin embargo, también se puede usar GAMS. 
Cuadro 4. Varianza observada en la producción tomando como base la matriz de 1993

\begin{tabular}{lccccccc}
\hline Año & $\begin{array}{c}\text { Baja } \\
\text { California }\end{array}$ & Sonora & Chihuahua & Coahuila & Nuevo León & Tamaulipas & $\begin{array}{c}\text { Frontera } \\
\text { Norte }\end{array}$ \\
\hline 1993 & 67.23 & 25.41 & 32.55 & 17.40 & 22.29 & 25.02 & 20.23 \\
1994 & 70.15 & 26.92 & 32.26 & 16.81 & 22.42 & 26.41 & 20.57 \\
1995 & 76.20 & 30.33 & 33.90 & 20.06 & 20.12 & 24.87 & 20.22 \\
1996 & 79.46 & 28.12 & 38.20 & 26.12 & 25.23 & 27.67 & 24.29 \\
1997 & 83.26 & 34.47 & 39.01 & 27.18 & 28.32 & 27.52 & 26.80 \\
1998 & 78.81 & 36.09 & 39.10 & 26.71 & 27.38 & 27.42 & 26.33 \\
1999 & 78.61 & 35.12 & 40.16 & 25.26 & 26.60 & 27.81 & 26.83 \\
2000 & 84.78 & 41.66 & 44.91 & 23.22 & 29.76 & 29.13 & 29.86 \\
2001 & 79.81 & 36.78 & 42.49 & 21.47 & 27.41 & 25.86 & 27.30 \\
2002 & 76.06 & 29.91 & 39.94 & 22.67 & 26.71 & 27.29 & 26.18 \\
2003 & 78.91 & 26.89 & 42.83 & 24.59 & 27.08 & 26.96 & 26.71 \\
2004 & 82.23 & 29.88 & 43.16 & 25.69 & 29.30 & 28.26 & 27.92 \\
2005 & 84.96 & 31.18 & 45.12 & 23.98 & 28.96 & 29.54 & 28.17 \\
2006 & 83.11 & 43.23 & 46.37 & 24.08 & 30.49 & 26.63 & 28.61 \\
R & 0.71 & 0.30 & 0.78 & 0.91 & 0.88 & -0.05 & 0.95 \\
\hline
\end{tabular}

Fuente: Elaboración propia.

Cuadro 5. Varianza observada en la producción tomando como base la MIP del año 2003

\begin{tabular}{cccccccc}
\hline Año & $\begin{array}{c}\text { Baja } \\
\text { California }\end{array}$ & Sonora & Chihuahua & Coahuila & $\begin{array}{c}\text { Nuevo } \\
\text { León }\end{array}$ & Tamaulipas & $\begin{array}{c}\text { Frontera } \\
\text { Norte }\end{array}$ \\
\hline 1993 & 108.80 & 31.50 & 63.81 & 23.90 & 32.78 & 29.93 & 26.97 \\
1994 & 112.58 & 34.00 & 63.73 & 22.84 & 33.53 & 29.08 & 27.51 \\
1995 & 121.87 & 41.81 & 72.94 & 27.85 & 30.98 & 22.60 & 27.76 \\
1996 & 121.41 & 36.27 & 71.02 & 36.79 & 36.33 & 25.47 & 32.49 \\
1997 & 122.98 & 46.02 & 68.44 & 37.86 & 40.07 & 28.14 & 35.71 \\
1998 & 123.07 & 48.24 & 67.67 & 37.49 & 39.96 & 31.97 & 35.61 \\
1999 & 121.34 & 44.57 & 63.57 & 35.51 & 38.82 & 36.30 & 35.84 \\
2000 & 126.65 & 53.71 & 68.73 & 32.27 & 42.34 & 35.19 & 39.11 \\
2001 & 118.76 & 46.50 & 64.58 & 29.85 & 40.18 & 41.91 & 36.25 \\
2002 & 114.29 & 36.50 & 62.27 & 31.73 & 39.37 & 36.69 & 34.76 \\
2003 & 114.93 & 31.20 & 65.24 & 34.96 & 39.90 & 41.11 & 35.36 \\
2004 & 119.01 & 34.00 & 66.67 & 36.57 & 43.14 & 40.30 & 37.19 \\
2005 & 122.32 & 36.52 & 72.50 & 33.59 & 41.57 & 43.26 & 37.69 \\
2006 & 120.81 & 56.96 & 83.37 & 33.65 & 42.58 & 33.73 & 38.84 \\
$R$ & 0.73 & 0.50 & -0.04 & 0.92 & 0.90 & 0.53 & 0.95 \\
\hline
\end{tabular}

Fuente: Elaboración propia 
De manera general, los resultados permiten apreciar que tanto en las entidades de la región por separado, como en la frontera norte en su conjunto, la varianza de la producción mostró un incremento para el periodo de estudio. El coeficiente $\mathrm{R}$ muestra que el aumento en la volatilidad se encuentra positiva y altamente correlacionado con el grado de concentración de la actividad productiva dentro del sector manufacturero, que en estas entidades se localiza principalmente en las actividades exportadoras. No obstante, la magnitud de este indicador y sus diferencias por estado, son sensibles a la matriz insumo-producto que se utiliza.

Las estimaciones muestran que Nuevo León, Coahuila y Tamaulipas, son las entidades con una menor varianza estimada y en consecuencia con una menor variabilidad en la producción, lo que estaría implicando que se encuentran mejor posicionadas para absorber los cambios en las condiciones económicas externas. Caso contrario sería el de Baja California, Chihuahua y Sonora.

Cuando se analiza la varianza de la región se observa que ésta aumenta pero también que los valores se incrementan significativamente entre la MIP de 1993 y la de 2003, lo cual sugiere cambios importantes en los coeficientes regionales de comercio. En 1993 los valores son para cada mip 20.23 y 26.97, mientras que en 2006 son 28.61 y 38.84 , es decir, hay diferencias de 33.3 y $35.8 \%$ respectivamente que reflejan la magnitud del rompimiento en los encadenamientos productivos locales, tan frecuentemente referenciados en los análisis del México posapertura. Si se consideran los años extremos en cada MIP se tiene que en la de 1993 se pasa de 20.23 a 28.61 y en la de 2003 , de 26.97 a 38.84, lo cual representa variaciones de 41.4 y $44 \%$ que pueden atribuirse a cambios en la demanda final relacionados con el aumento en la volatilidad por la mayor concentración de la actividad productiva. Ambos tipos de movimientos representan un aumento en la volatilidad que fluctúa entre 75 y $80 \%$ para la región en su conjunto.

La Gráfica 3 muestra la varianza observada para la frontera norte con cada una de las cuatro matrices utilizadas. Se puede apreciar que independientemente de la matriz utilizada, la tendencia es claramente ascendente.

Baja California es la entidad que muestra una mayor volatilidad en su crecimiento pues la varianza observada en el año de 2003 con la MIP2003 es la más alta con un valor de 120.81 . El aumento en la volatilidad debido al rompimiento de los encadenamientos productivos locales es de $61.83 \%$ y se obtiene comparando el valor observado para la varianza en el año 1993 con las mip 1993 y MIP2003 (67.23 y 108.80). Por su parte, el incremento explicado por las variaciones en la demanda final, comparando la varianza para 2006 con la de 1993 ambas con la MIP2003, representa el 11.04\%. La suma de ambas magnitudes arroja un incremento de la volatilidad equivalente al $72.87 \%$. 
Gráfica 3. Varianza de la producción en la Frontera Norte de México, 1993-2006

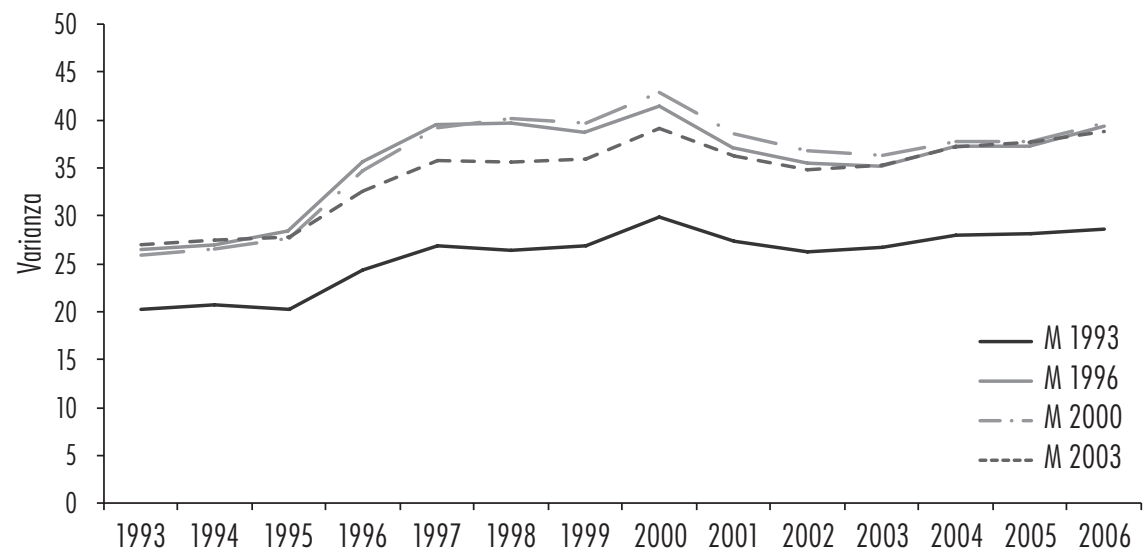

Fuente: Elaboración propia.

El coeficiente de correlación ( $\mathrm{R}$ ) de 0.73 , refleja un alto grado de asociación positiva entre la volatilidad de la tasa de crecimiento y la concentración de la producción en el sector manufacturero, mientras que la tendencia creciente de la varianza, muestra que la estructura de la producción en la entidad es similar a la de 1993 sólo que a un nivel más elevado de volatilidad, como se aprecia en la Gráfica 4.

Gráfica 4. Varianza de la producción en Baja California, 1993-2006

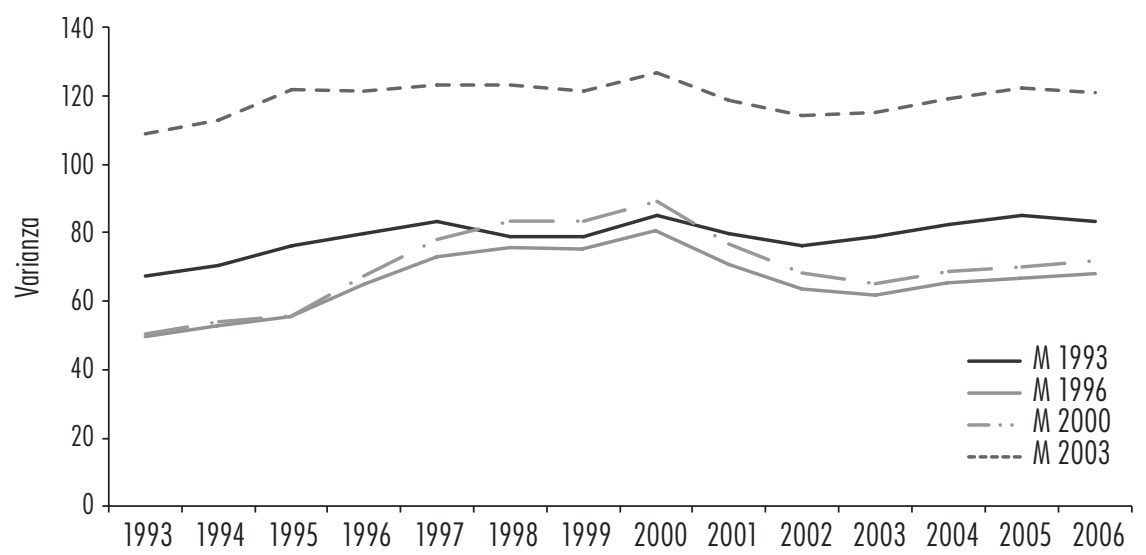

Fuente: Elaboración propia. 
En la Gráfica 5 se aprecia cómo evoluciona la varianza para el caso de Sonora mostrando una tendencia ascendente con un repunte muy pronunciado en los años recientes. Sonora es una de las entidades en donde la volatilidad sufre un incremento mayor, lo que se explica por la ruptura de los encadenamientos productivos locales en $24 \%$ y por los cambios en su estructura industrial en $81 \%$. El aumento total de la volatilidad alcanzó el 105\%. Aunque en Sonora el coeficiente $\mathrm{R}$ es de los menores, éste muestra una asociación positiva e incrementa de 0.30 con la MIP1993 a 0.50 con la MIP2003 que refleja un aumento en la relación entre volatilidad y concentración productiva en la industria manufacturera.

Gráfica 5. Varianza de la producción en Sonora, 1993-2006

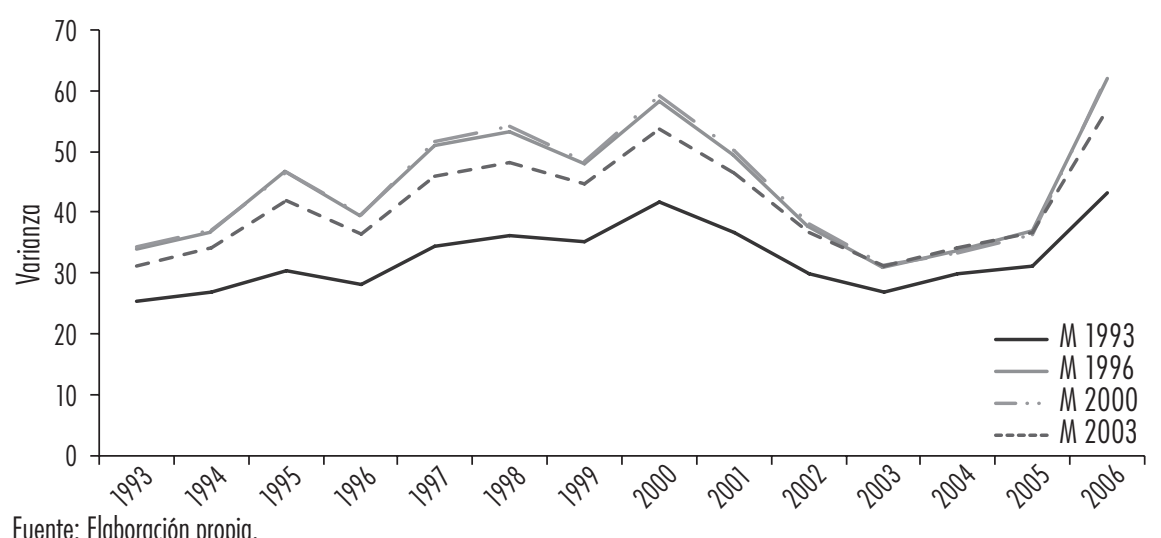

Fuente: Elaboración propia.

El estado de Chihuahua es el que muestra el mayor aumento de volatilidad en su crecimiento con $126 \%$ del cual, en sentido contrario a Sonora, el $97 \%$ se explica por la ruptura de los encadenamientos productivos locales y el 51\% refleja los cambios en la estructura de su producción. Con respecto al coeficiente $\mathrm{R}$, éste tiene un comportamiento atípico, pues si bien con la MIP1993 su valor es 0.78 , con la MIP2003 se vuelve no significativo y pasa a -0.04. Este resultado se debe a que la MIP2003 identifica a los sectores comercio, restaurantes y hoteles, y a la minería como las fuentes de inestabilidad, no a la industria manufacturera ${ }^{4}$ (ver Gráfica 6).

En el caso de Coahuila, la variabilidad del crecimiento ha mostrado un aumento de $37 \%$ imputable a los cambios en los coeficientes regionales de comercio

4 El coeficiente de correlación entre la varianza y el HHI para el total de la actividad económica es de 0.44 en la entidad. 
Gráfica 6. Varianza de la producción en Chihuahua, 1993-2006

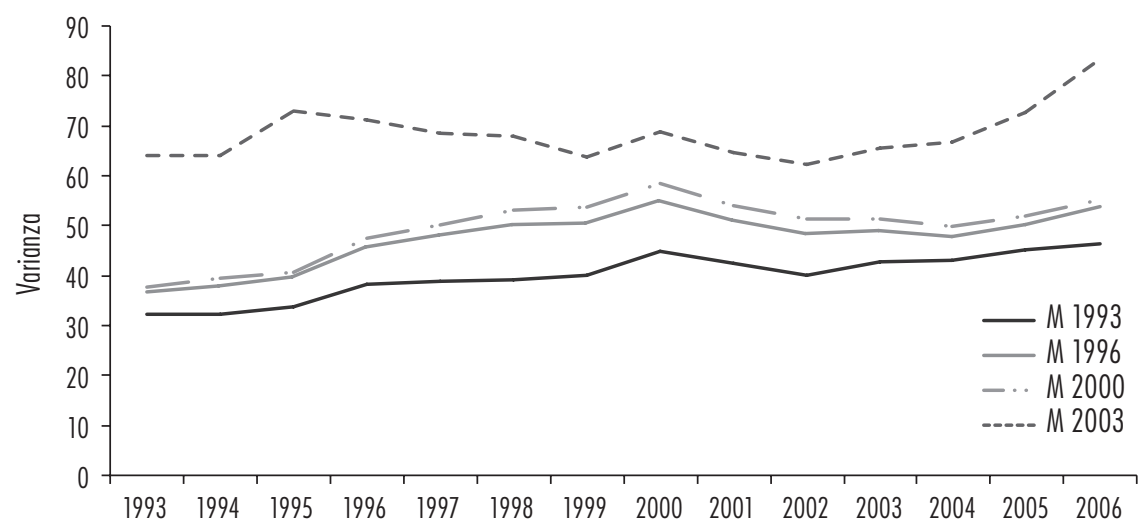

Fuente: Elaboración propia.

y de $41 \%$ como consecuencia de los cambios en su estructura industrial, con lo que se tiene un aumento general de 78\%. El coeficiente de correlación entre volatilidad y estructura industrial es de 0.92 , sólo menor que el de la frontera norte en su conjunto. También en una situación atípica con relación a las demás entidades, en este caso la serie de la varianza evaluada con la MIP2003 se encuentra claramente por debajo de las evaluadas con la MIP1996 y MiP2000, indicando que la estructura industrial de la entidad vigente para el año 2003 es en general más estable que la prevaleciente durante el periodo 1996-2002 (ver Gráfica 7).

Gráfica 7. Varianza de la producción en Coahuila, 1993-2006

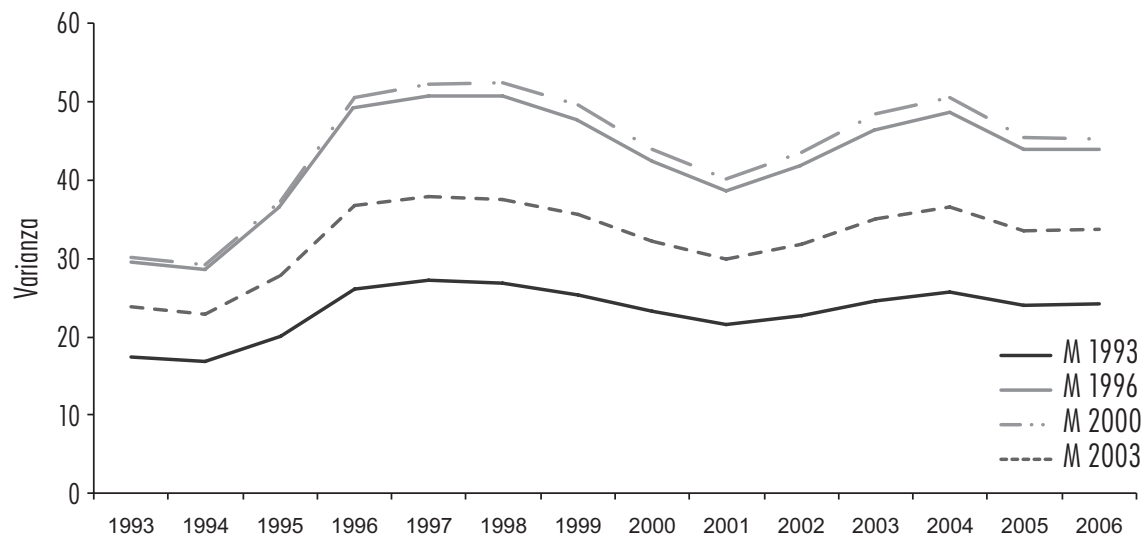

Fuente: Elaboración propia. 
La entidad que muestra un comportamiento más cercano al de la frontera norte como región en lo que se refiere a volatilidad, es Nuevo León, tal como se puede apreciar en la Gráfica 8. En este caso el aumento de la varianza atribuible a la ruptura de los encadenamientos productivos representa el $47 \%$ mientras que el imputable a los cambios en el patrón de especialización es del 30\% para un incremento total de $77 \%$. Nuevo León también muestra un alto coeficiente de correlación de 0.90 , indicando una elevada correspondencia entre el nivel de volatilidad y el patrón de especialización manufacturero que ha seguido esta entidad.

Gráfica 8. Varianza de la producción en Nuevo León, 1993-2006

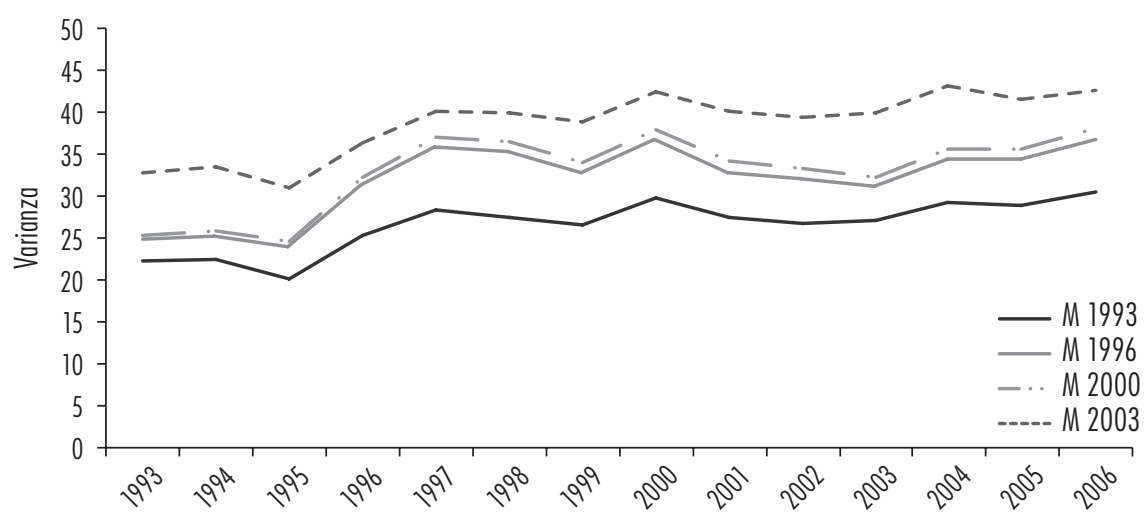

Fuente: Elaboración propia.

Tamaulipas es la entidad que muestra tanto un menor nivel de volatilidad como los menores incrementos a lo largo del periodo de estudio. Así, la variación correspondiente a cambios en los coeficientes regionales de comercio es de $19.61 \%$ y la correspondiente a los cambios en el patrón de producción es de $12.69 \%$, para un total de $32.30 \%$. No obstante lo anterior, en este caso también se observan cambios pronunciados en el nivel de variabilidad de un año a otro como se ilustra en la Gráfica 9. El coeficiente de correlación se comporta en sentido contrario al de Chihuahua, pues no es significativo con la MIP 1993 con un valor de -0.05 , y de 0.53 con la MIP2003, ilustrando la importancia adquirida por el sector manufacturero como determinante de la inestabilidad.

Adicionalmente y de manera general se pueden apuntar las siguientes observaciones con respecto a la evolución de la volatilidad económica en la región: 1) Independientemente de la MIP que se utilice para evaluar la varianza, con la excepción de Tamaulipas, en el resto de las entidades la volatilidad muestra una 
Gráfica 9. Varianza de la producción en Tamaulipas, 1993-2006

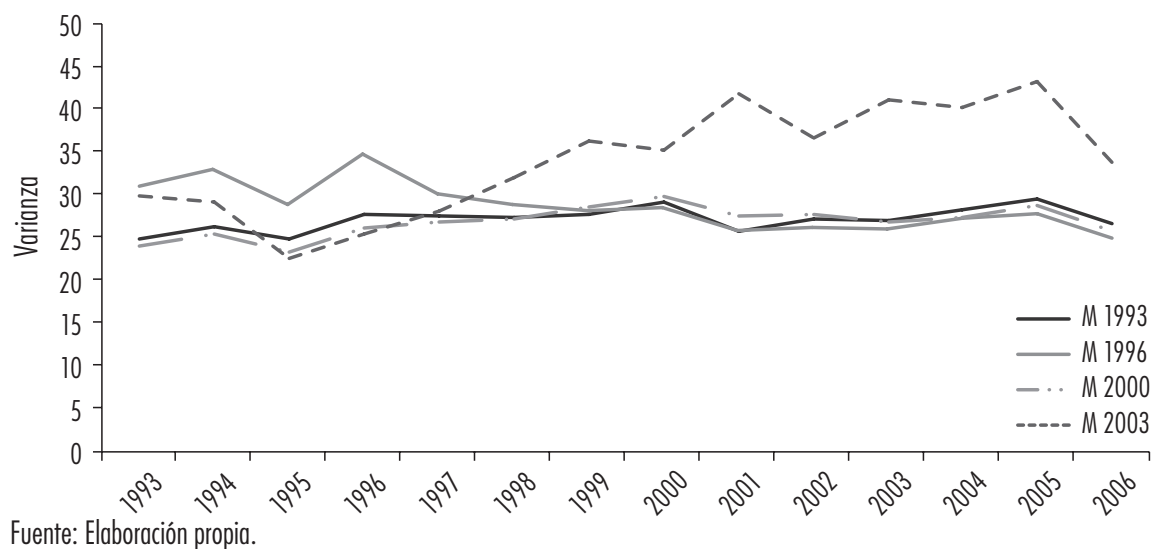

tendencia creciente. 2) En todas las gráficas se observa un punto de inflexión en el año 2000 que responde a la desaceleración económica en Estados Unidos y la consecuente reducción de las exportaciones hacia ese país. 3) Los años de mayor inestabilidad son diferentes para cada una de las entidades analizadas, siendo 2006 para Sonora y Chihuahua, 2000 para Baja California, 1997 para Coahuila, 2004 para Nuevo León y 2005 para Tamaulipas. En promedio el pico más alto a nivel regional se alcanza en 2000. Esto sugiere que se trata de una región en donde cada entidad mantiene sus especificidades y es afectada de manera diferenciada por los fenómenos económicos externos e internos.

\section{IMPLICACIONES DE POLÍTICA INDUSTRIAL}

El instrumental analítico utilizado en este trabajo permite aprovechar la información generada para proponer cambios en la estructura industrial que podrían contribuir a la construcción de una economía más estable. Se muestran los resultados de un ejercicio en donde se calculan los cambios en la participación de los distintos sectores que permiten minimizar la varianza en la tasa de crecimiento de la producción. Valores positivos indican que el sector correspondiente reduce la varianza mientras que valores negativos indican lo contrario.

En Baja California se identifican siete sectores cuya expansión reduce la varianza de la producción agregada, mientras que otros diez la aumentan (ver Cuadro 6). Aunque los valores que se presentan en el cuadro indican en cuánto debe crecer la participación de un sector en la DF para minimizar la varianza, no 
se muestra cuáles contribuyen más en su reducción. En consecuencia, se realiza un ejercicio adicional para identificar a aquellos que ofrecen una mayor aportación en este sentido.

En Baja California los sectores que más potencial tienen para contribuir a un crecimiento estable son el 17 (Otros servicios), seguido por 1 (Agricultura,

Cuadro 6. Cambios en la estructura productiva para aumentar la estabilidad en la tasa de crecimiento Matriz de 2003

\begin{tabular}{|c|c|c|c|c|c|c|c|}
\hline Concepto & $\begin{array}{c}\text { Baja } \\
\text { California }\end{array}$ & Sonora & Chihuahua & Coahuila & $\begin{array}{l}\text { Nuevo } \\
\text { León }\end{array}$ & Tamaulipas & $\begin{array}{c}\text { Frontera } \\
\text { Norte }\end{array}$ \\
\hline 1 Agricultura, silvicultura y pesca & 3311.2 & 309.6 & -276.5 & 761.2 & -121.6 & 81.9 & -559.7 \\
\hline 2 Minería & 49156.5 & -181.4 & -430.3 & 241.4 & 2007.1 & -43.1 & -179.9 \\
\hline $\begin{array}{l}3 \text { Productos alimenticios, } \\
\text { bebidas y tabaco }\end{array}$ & 552.1 & 210.2 & 539.9 & 421.8 & 124.1 & 341.6 & -48.0 \\
\hline $\begin{array}{l}4 \text { Textiles, prendas de vestir e } \\
\text { industria del cuero }\end{array}$ & -2257.6 & 632.2 & -212.1 & -1582.9 & 2724.7 & 502.1 & -1233.0 \\
\hline $\begin{array}{l}5 \text { Industria de la madera y } \\
\text { productos de madera }\end{array}$ & 1790.2 & 2896.7 & -856.2 & -46294.0 & 274.5 & 6744.1 & -668.4 \\
\hline $\begin{array}{l}6 \text { Papel, productos de papel, } \\
\text { imprenta e industria editorial }\end{array}$ & 1305.4 & -4123.5 & -5691.7 & -13162.4 & -3457.1 & 2568.0 & -7365.7 \\
\hline $\begin{array}{l}7 \text { Sustancias químicas y } \\
\text { derivados del petróleo }\end{array}$ & -328.7 & -1481.1 & -2337.9 & 167.3 & 305.3 & -250.7 & -86.8 \\
\hline $\begin{array}{l}8 \text { Productos de minerales no } \\
\text { metálicos }\end{array}$ & 2125.9 & -953.3 & 904.3 & 1510.6 & 1827.1 & -374.6 & 1189.3 \\
\hline 9 Industrias metálicas básicas & -4827.4 & 281.5 & -2559.9 & 288.8 & 89.2 & 3756.0 & 264.3 \\
\hline $\begin{array}{l}10 \text { Productos metálicos, } \\
\text { maquinaria y equipo }\end{array}$ & -120.3 & -58.4 & -10.1 & -175.0 & -29.6 & -92.6 & -62.4 \\
\hline $\begin{array}{l}11 \text { Otras industrias } \\
\text { manufactureras }\end{array}$ & -367.8 & 379.5 & 2418.5 & -13106.1 & -125.1 & -384.2 & 2826.6 \\
\hline 12 Construcción & -620.6 & -52.7 & 271.3 & 427.4 & 516.9 & 28.8 & -227.1 \\
\hline 13 Electricidad, gas y agua & -174.0 & -468.7 & -1440.8 & 1829.1 & -2534.3 & 252.1 & -421.9 \\
\hline $\begin{array}{l}14 \text { Comercio, restaurantes y } \\
\text { hoteles }\end{array}$ & -25.2 & -118.7 & -285.2 & 151.6 & -156.2 & -195.6 & -165.7 \\
\hline $\begin{array}{l}15 \text { Transporte, almacenaje y } \\
\text { comunicaciones }\end{array}$ & -14.7 & -167.2 & -0.5 & 198.1 & -644.6 & -112.1 & -172.8 \\
\hline $\begin{array}{l}16 \text { Servicios financieros, } \\
\text { seguros, alquiler y bienes } \\
\text { inmuebles }\end{array}$ & -88.4 & 84.6 & 687.9 & -74.9 & 304.6 & 63.0 & 507.0 \\
\hline 17 Otros Servicios & 99.7 & 167.7 & 465.2 & 299.4 & 40.5 & 148.8 & 412.7 \\
\hline
\end{tabular}

Fuente: Elaboración propia.

Nota: El valor indica en cuánto debe crecer la participación del sector en la DF para minimizar la varianza. 
silvicultura y pesca) y el 3 (Productos alimenticios, bebidas y tabaco). Por su parte, los que más inestabilidad generan son el 14 (Comercio, restaurantes y hoteles), el 10 (Productos metálicos, maquinaria y equipo) y el 7 (Sustancias químicas y derivados del petróleo). Es importante destacar que el primer grupo ha disminuido notablemente su participación en el PIB de la entidad, siendo la caída más pronunciada la del sector 3 cuya producción pasó de 20.7\% del total de las manufacturas en 1993, a 14.2\% en 2006. Aunque menor, los sectores 17 y 1 también tuvieron una contracción relativa al caer su participación de $22.2 \%$ a $20.9 \%$ y de $3.7 \%$ a $1.3 \%$ con respecto al total de la actividad económica respectivamente. Lo contrario ocurrió con los sectores que generan inestabilidad, pues éstos han aumentado su participación en el producto: el sector 14 pasó de $23.9 \%$ en 1993 a $29 \%$ en 2003 , el 10 de $43.4 \%$ a $51.7 \%$ y el 7 de $4.8 \%$ a $5.2 \%$.

La tendencia observada en Baja California se repite en el resto de las entidades en el sentido de que los sectores que reducen la inestabilidad contraen su participación en la producción, mientras que aquéllos con potencial para aumentarla tienden a expandirse. Esto propicia una descompensación en la estructura productiva y sugiere la necesidad de impulsar cambios estructurales para favorecer la estabilidad.

Ahora bien, aunque es cierto que los sectores que se deben impulsar son los que han ido perdiendo participación en el pIB a lo largo de las últimas dos décadas, tiene sentido apoyarlos si se consideran los beneficios que se obtendrían en materia de estabilidad económica y porque debido a su alto grado de orientación hacia el mercado interno fortalecerían este segmento de nuestra economía tan castigado en años recientes, contribuyendo a restaurar los eslabones de la cadena productiva dentro de la industria nacional. El impulso a dichos sectores es consistente con el planteamiento de Dussel y Katz (2002), Ocegueda (2005 y 2006), Dávila (2004), entre otros, que alertan sobre la necesidad de fortalecer los encadenamientos productivos locales y contrarrestar el proceso de maquilización que se da principalmente en la frontera norte de México, así como de estimular el desarrollo de los sectores orientados hacia el mercado interno con base en las características específicas de cada región.

A continuación se presentan los resultados de un ejercicio de simulación considerando dos escenarios: $\boldsymbol{a}$ ) se supone que las entidades de la región continúan con la misma tendencia en su patrón de especialización y; $\boldsymbol{b}$ ) se asume una tasa de crecimiento necesaria en los sectores estratégicos, en este caso los que contribuyen a la estabilidad, para propiciar un retorno a los niveles de estabilidad previos a la apertura comercial imponiendo como restricción que la tasa de crecimiento del conjunto de la economía no puede ser inferior a la actual. 
Juan Manuel Ocegueda, Antonio Escamilla y Alejandro Mungaray

Cuadro 7. Varianza de la producción estimada permitiendo que las entidades sigan con su patrón de especialización

\begin{tabular}{cccccccc}
\hline Año & $\begin{array}{c}\text { Baja } \\
\text { California }\end{array}$ & Sonora & Chihuahua & Coahuila & Nuevo León & Tamaulipas & $\begin{array}{c}\text { Frontera } \\
\text { Norte }\end{array}$ \\
\hline 2007 & 121.87 & 60.54 & 73.36 & 34.73 & 43.74 & 39.73 & 39.61 \\
2008 & 122.74 & 64.19 & 70.10 & 36.01 & 44.65 & 39.39 & 40.33 \\
2009 & 124.04 & 68.14 & 69.77 & 37.38 & 45.77 & 39.50 & 41.34 \\
2010 & 125.09 & 72.93 & 70.29 & 38.07 & 46.56 & 39.43 & 42.14 \\
2011 & 125.62 & 77.00 & 71.22 & 38.58 & 47.04 & 39.28 & 42.75 \\
2012 & 126.05 & 81.72 & 72.07 & 39.17 & 47.54 & 39.08 & 43.39 \\
\hline
\end{tabular}

Fuente: Elaboración propia

Cuadro 8. Varianza de la producción estimada propiciando un crecimiento adicional en la demanda final en los sectores estratégicos

\begin{tabular}{cccccccc}
\hline Año & $\begin{array}{c}\text { Baja } \\
\text { California } \\
(10 \%)\end{array}$ & $\begin{array}{c}\text { Sonora } \\
(12 \%)\end{array}$ & $\begin{array}{c}\text { Chihuahua } \\
(8 \%)\end{array}$ & $\begin{array}{c}\text { Coahuila } \\
(9 \%)\end{array}$ & $\begin{array}{c}\text { Nuevo León } \\
(8 \%)\end{array}$ & $\begin{array}{c}\text { Tamaulipas } \\
(8 \%)\end{array}$ & $\begin{array}{c}\text { Frontera } \\
\text { Norte } \\
(10 \%)\end{array}$ \\
\hline 2007 & 119.63 & 55.74 & 71.86 & 33.08 & 42.30 & 38.43 & 38.19 \\
2008 & 118.09 & 54.02 & 67.18 & 32.54 & 41.71 & 36.75 & 37.35 \\
2009 & 117.85 & 52.37 & 65.40 & 32.03 & 41.31 & 35.51 & 36.76 \\
2010 & 117.51 & 51.44 & 64.82 & 30.91 & 40.59 & 34.17 & 35.88 \\
2011 & 115.87 & 49.73 & 64.25 & 29.70 & 39.53 & 32.80 & 34.76 \\
2012 & 114.17 & 48.32 & 63.48 & 28.60 & 38.48 & 31.49 & 33.60 \\
\hline
\end{tabular}

Fuente: Elaboración propia

Como se puede observar el impulso a los sectores estratégicos de acuerdo con los Cuadros 7 y 8 permite disminuir de manera consistente la volatilidad del crecimiento económico de cada una de las entidades consideradas y de la región en su conjunto. Adicionalmente, este proceso de diversificación no implicaría una disminución en la tasa de crecimiento tanto de la demanda final, como del valor bruto de la producción en ninguno de los casos considerados. Este ejercicio muestra de manera hipotética cómo una diversificación sectorial dentro de la planta productiva puede generar mayor estabilidad sin empeorar el desempeño económico actual, pero lo más importante quizá, es que sugiere la factibilidad de lograr lo mismo aumentando la tasa de crecimiento si se realiza la reestructuración sectorial adecuada. De acuerdo con este ejercicio los sectores que la política industrial debe impulsar en la frontera norte para ganar estabilidad son el 
$1,3,16$ y 17 , lo cual no se contrapone con la idea de que se sigan desarrollando aquéllos que logran sobrevivir a través del mercado.

Un aspecto importante que destaca en este análisis es la necesidad de replantear el modelo de desarrollo agropecuario, pues como se ha señalado, éste resulta estratégico en el sentido mencionado en este trabajo, en la mayoría de las entidades sólo con la excepción de Chihuahua y Nuevo León.

\section{CONCLUSIONES}

Los resultados obtenidos en este trabajo indican que de 1993 a 2006 se incrementa la volatilidad de la tasa de crecimiento económico en las entidades de la frontera norte de México como consecuencia de una alta concentración de la actividad productiva en la industria manufacturera. El proceso de cambio estructural al concentrar la producción en un reducido grupo de industrias exportadoras, ha vuelto más inestable el desempeño económico de la región, haciéndolo más susceptible a las fluctuaciones externas, por lo que la idea de recuperar la importancia del mercado interno ha ido adquiriendo cada vez mayores adeptos. Los ejercicios presentados muestran que es posible tener un desempeńo superior al actual o, en el peor de los casos similar, reduciendo la vulnerabilidad a los ciclos externos si se impulsa un conjunto de sectores, muchos de ellos de corte tradicional, con fuerte impacto en los mercados locales y regionales. Si bien una afirmación como ésta pareciera contraponerse a la corriente dominante dentro de la literatura actual que pugna por orientar la especialización hacia actividades modernas con alto contenido tecnológico y uso intensivo de capital humano, en México algunos de estos sectores no están ejerciendo el liderazgo que de ellos se esperaría (Ocegueda, Varela y Castillo, 2009), en parte por fallas en las políticas destinadas a incentivarlos; porque se trata de actividades modernas que sólo desplazan a nuestro país los procesos intensivos en mano de obra; o por la persistencia de un segmento aún muy amplio de empresas micro, pequeñas y medianas que operan con tecnologías tradicionales y que difícilmente podrán reconvertirse en el mediano plazo. No obstante, estos sectores pueden jugar un rol relevante en el fortalecimiento del mercado interno y en la reducción de la vulnerabilidad externa si se establecen políticas adecuadas para su desarrollo y modernización.

Los sectores con mayor potencial para contribuir a un crecimiento estable (otros servicios; agricultura, silvicultura y pesca; productos alimenticios, bebidas y tabaco), aglutinan a un número importante de micros y pequeñas empresas informales, en donde una política industrial orientada a desarrollarlas, a través 
de créditos blandos provenientes de fondos públicos, asistencia técnica universitaria y facilidades para su formalización, podrían generar, además de mayor estabilidad ante las fluctuaciones económicas externas, posibilidades de ascenso social para grupos que actualmente viven en condiciones de marginalidad; desarrollo de habilidades empresariales que podrían potenciar y dinamizar la economía, así como una mayor captación de impuestos al incorporar a un amplio sector de la sociedad a la economía formal. En una estrategia de esta naturaleza es muy importante que las acciones de política gubernamental se complementen con la participación comprometida de las universidades que pueden tener una importante contribución, proporcionando recursos humanos para la asistencia técnica mediante el servicio social obligatorio.

La búsqueda de un modelo de especialización o de reorganización de la estructura productiva que mejore el desempeńo económico a largo plazo, debe considerar además de los criterios tradicionales asociados con la nueva teoría del crecimiento y la teoría poskeynesiana, elementos adicionales como la variabilidad en la tasa de crecimiento del producto sectorial, el mercado al que se orienta la producción, el nivel de encadenamientos productivos locales que genera y la dotación de recursos naturales de cada región.

Una economía que concentra sus actividades productivas en pocos sectores es más vulnerable a las fluctuaciones del ciclo económico y está sujeta como resultado de ello a una mayor inestabilidad. Este riesgo se puede controlar o por lo menos disminuir promoviendo la diversificación de la estructura productiva. Así, una trayectoria de crecimiento estable se puede construir alentando una estructura económica que combine sectores de lento y rápido crecimiento como ocurre en la teoría del portafolio, en donde la cartera óptima corresponde a aquella que diversifica el riesgo mediante la combinación de activos con distintos rendimientos y, por tanto, con diversos niveles de riesgo.

\section{REFERENCIAS BIBLIOGRÁFICAS}

Castillo, Ramón A., Rogelio Varela y Juan Manuel Ocegueda, "Synchronization of Economic Activity Between Mexico and the U.s.: How did it Happen?", en Revista de Análisis Económico, Vol. 25(1), Santiago, ILADEs/Georgetown University, junio de 2010, pp. 15-48.

Conroy, M.E., "The Concept and Measurement of Regional Industrial Diversification", en Southern Economic Journal, Vol. 41, núm. 3, Richmond, Allen Press Association, enero de 1975, pp. 492-505. 
Dávila, Alejandro, "México: concentración y localización del empleo manufacturero, 1980-1998”, en Economía Mexicana, Nueva Época, Vol. xIII, núm 2, México, CIDE, segundo semestre de 2004, pp. 209-254.

, "Matriz de insumo-producto de la economía de Coahuila e identificación de sus flujos intersectoriales más importantes", en Economía Mexicana, Nueva Época, Vol. xI, núm 1, México, CIDE, primer semestre de 2002, pp. 79-117.

Dussel, Enrique y Jorge Katz, Diferentes estrategias en el nuevo modelo económico latinoamericano: importaciones temporales para su reexportación y transformación de materias primas, Santiago, CEPAL, septiembre 2002.

Flegg, A.T. y C.D. Webber, "On the Appropriate use of Location Quotients in Generating Regional Input-output Tables”, en Regional Studies, Routledge, Vol. 31, núm. 8, Seaford, Routledge, noviembre de 1997, pp. 795-805.

Flegg, A.T., C.D. Webber y M. Elliott, "On the Appropriate use of Location Quotients in Generating Regional Input-output Tables: Reply”, en Regional Studies, Vol. 29, núm. 6, Seaford, Routledge, abril de 1995, pp. 547-561.

Fuentes, N.A., "Construcción de una Matriz Regional de Insumo Producto", en Problemas del Desarrollo, Vol. 36, núm. 140, México, IIEc-unam, eneromarzo de 2005, pp. 90-112.

Haugen, Robert A., Modern Investment Theory, New Jersey, Prentice Hall, 1997. Koren, Miklós y Silvana Tenreyro, "Volatility and Development", Discussion Paper, núm. 706, Centre for Economic Performance, London School of Economics and Political Science, 2005.

Lucas, Robert, "On the Mechanics of Economic Development", en Journal of Monetary Economics, Vol. 22, núm. 1, Rochester, Elsevier, julio de 1988, pp. $3-42$.

Ocegueda, Juan Manuel, "Especialización productiva y crecimiento económico", Alejandro Mungaray y Juan Manuel Ocegueda (Eds.), Estudios económicos sobre Baja California, Porrúa-Universidad Autónoma de Baja California, 2006.

, "Comercio y crecimiento económico en Baja California", en Investigación Económica, Vol. 64, núm. 251, FE-UNAM, enero-marzo 2005, pp. 111-139.

, Ramón Castillo y Rogelio Varela, "Crecimiento regional en México. Especialización y sectores clave”, en Problemas del Desarrollo, Vol. 40, núm. 159, México, IIEc-UNAM, octubre-diciembre, 2009, pp. 61-84.

Siegel, Paul B., Thomas G. Johnson y Jeffrey Alwang, "Regional Economic Diversity and Diversification", en Growth and Change, Vol. 26, núm. 2, Malden, John Wiley and Sons, primavera de 1995, pp. 261-284. 